\title{
Analysis of sleep disorders under pain using an optogenetic tool: possible involvement of the activation of dorsal raphe nucleus-serotonergic neurons
}

\author{
Hisakatsu Ito ${ }^{1,2}$, Makoto Yanase ${ }^{1}$, Akira Yamashita ${ }^{1}$, Chigusa Kitabatake ${ }^{1}$, Asami Hamada', Yuki Suhara',
} Michiko Narita ${ }^{1}$, Daigo Ikegami ${ }^{1}$, Hiroyasu Sakai ${ }^{1}$, Mitsuaki Yamazaki ${ }^{2^{*}}$ and Minoru Narita ${ }^{1,2^{*}}$

\begin{abstract}
Background: Several etiological reports have shown that chronic pain significantly interferes with sleep. Inadequate sleep due to chronic pain may contribute to the stressful negative consequences of living with pain. However, the neurophysiological mechanism by which chronic pain affects sleep-arousal patterns is as yet unknown. Although serotonin $(5-H T)$ was proposed to be responsible for sleep regulation, whether the activity of 5-HTergic neurons in the dorsal raphe nucleus (DRN) is affected by chronic pain has been studied only infrequently. On the other hand, the recent development of optogenetic tools has provided a valuable opportunity to regulate the activity in genetically targeted neural populations with high spatial and temporal precision. In the present study, we investigated whether chronic pain could induce sleep dysregulation while changing the activity of DRN-5-HTergic neurons. Furthermore, we sought to physiologically activate the DRN with channelrhodopsin-2 (ChR2) to identify a causal role for the DRN-5-HT system in promoting and maintaining wakefulness using optogenetics.

Results: We produced a sciatic nerve ligation model by tying a tight ligature around approximately one-third to one-half the diameter of the sciatic nerve. In mice with nerve ligation, we confirmed an increase in wakefulness and a decrease in non-rapid eye movement (NREM) sleep as monitored by electroencephalogram (EEG). Microinjection of the retrograde tracer fluoro-gold (FG) into the prefrontal cortex (PFC) revealed several retrogradely labeled-cells in the DRN. The key finding of the present study was that the levels of 5-HT released in the PFC by the electrical stimulation of DRN neurons were significantly increased in mice with sciatic nerve ligation. Using optogenetic tools in mice, we found a causal relationship among DRN neuron firing, cortical activity and sleep-to-wake transitions. In particular, the activation of DRN-5-HTergic neurons produced a significant increase in wakefulness and a significant decrease in NREM sleep. The duration of NREM sleep episodes was significantly decreased during photostimulation in these mice.
\end{abstract}

Conclusions: These results suggest that neuropathic pain accelerates the activity of DRN-5-HTergic neurons. Although further loss-of-function experiments are required, we hypothesize that this activation in DRN neurons may, at least in part, correlate with sleep dysregulation under a neuropathic pain-like state.

Keywords: Optogenetics, Electroencephalogram, Dorsal raphe nucleus, Neuropathic pain, Sleep

\footnotetext{
* Correspondence: myama@med.u-toyama.ac.jp; narita@hoshi.ac.jp

${ }^{2}$ Department of Anesthesiology, Graduate School of Medical and

Pharmaceutical Sciences for Education, Toyama University, 2630 Sugitani,

Toyama 930-0194, Japan

'Department of Pharmacology, Hoshi University School of Pharmacy and

Pharmaceutical Sciences, 2-4-41 Ebara, Shinagawa-ku, Tokyo 142-8501, Japan

Full list of author information is available at the end of the article
}

\section{Biomed Central}

(C) 2013 Ito et al.; licensee BioMed Central Ltd. This is an Open Access article distributed under the terms of the Creative Commons Attribution License (http://creativecommons.org/licenses/by/2.0), which permits unrestricted use, distribution, and reproduction in any medium, provided the original work is properly cited. The Creative Commons Public Domain Dedication waiver (http://creativecommons.org/publicdomain/zero/1.0/) applies to the data made available in this article, unless otherwise stated. 


\section{Background}

It is generally acknowledged that neuropathic pain is extremely difficult to treat, and a major factor that affects outcomes is the presence of comorbidities, such as poor sleep and mood disorders. In clinical studies, most patients began to have difficulties with sleep after they began experiencing chronic pain $[1,2]$. However, the neurophysiological mechanism by which chronic pain affects sleep/arousal patterns is as yet unknown.

Serotonin (5-HT) was initially proposed to be responsible for the initiation and maintenance of sleep [3]. The firing rate of 5-HT-containing dorsal raphe nucleus (DRN) neurons decreases during slow wave sleep relative to that in wakefulness [4]. 5-HTergic neurons in the DRN fire tonically and regularly at $3-5 \mathrm{~Hz}$ in wakefulness, fire less during non-rapid eye movement (NREM) sleep, and are silent during rapid eye movement (REM) sleep [5-9]. Therefore, the DRN is a 5-HTergic brainstem structure that is thought to be important for promoting arousal.

Some neurons in the DRN respond to nociceptive stimuli. Noxious stimulation caused changes in the electrical activity of DRN neurons [10]. This concept was confirmed by the 2-deoxy-D-glucose [11] and c-fos [12] methods. However, whether the activity of 5-HTergic neurons in the DRN is affected by chronic pain has been studied only infrequently. In a previous study, 5-HT transporter expression was shown to be significantly increased in the DRN of sciatic nerve-ligated rats [13]. To investigate the influence of neuropathic pain on DRN 5HTergic neurons, we performed an in vivo dialysis study. We found that the 5-HT levels in the prefrontal cortex (PFC) were significantly increased in nerve-ligated mice compared to those in sham-operated mice after electrical stimulation of the DRN.

New tools are needed to selectively manipulate the discharge activities of the DRN in freely moving mice at time-scales that are relevant to natural sleep-wake events. The recent development of an optogenetic tool $[14,15]$ has provided a valuable opportunity to regulate the activity in genetically targeted neural populations with high spatial and temporal precision [16-19]. Optogenetics describes the now widespread use of microbial opsins [20] or related tools, that can be activated by illumination to manipulate cells even within intact tissue or behaving animals [21-23]. We stimulated DRN neurons with channelrhodopsin-2 (ChR2; a cation channel that is sensitive to $473 \mathrm{~nm}$ blue light) [17]. We found that mice showed an increase in the mean time of wakefulness, and decreases in the mean time and duration of NREM sleep, during optical stimulation of the DRN. To our knowledge, no previous reports have described the results obtained by stimulation of the DRN, which suggest a potential mechanism for the sleep disturbance induced by neuropathic pain.

\section{Results}

Thermal hyperalgesia induced by sciatic nerve ligation

Sciatic nerve ligation caused a marked decrease in the latency of paw withdrawal in response to thermal stimuli only on the ipsilateral side (Figure 1: thermal hyperalgesia, $\mathrm{p}<0.05$ vs. sham group).

\section{Changes in vigilance under a neuropathic pain-like state with EEG/EMG}

With this experimental model for neuropathic pain, we investigated the changes in sleep patterns. Frontal cortical activity and postural muscle tone, as monitored by EEG/ EMG, are useful for identifying sleep/wake abnormalities. Vigilance was classified offline into 3 stages: wakefulness, rapid eye movement sleep, and NREM sleep. Sciatic nerve ligation for 7 days was associated with statistically significant increases in wakefulness (Figure 2A: $\mathrm{p}<0.001$ vs. sham group). Sciatic nerve ligation mice showed a statistically significant decrease in NREM sleep (Figure 2B: $\mathrm{p}<0.01$ vs. sham

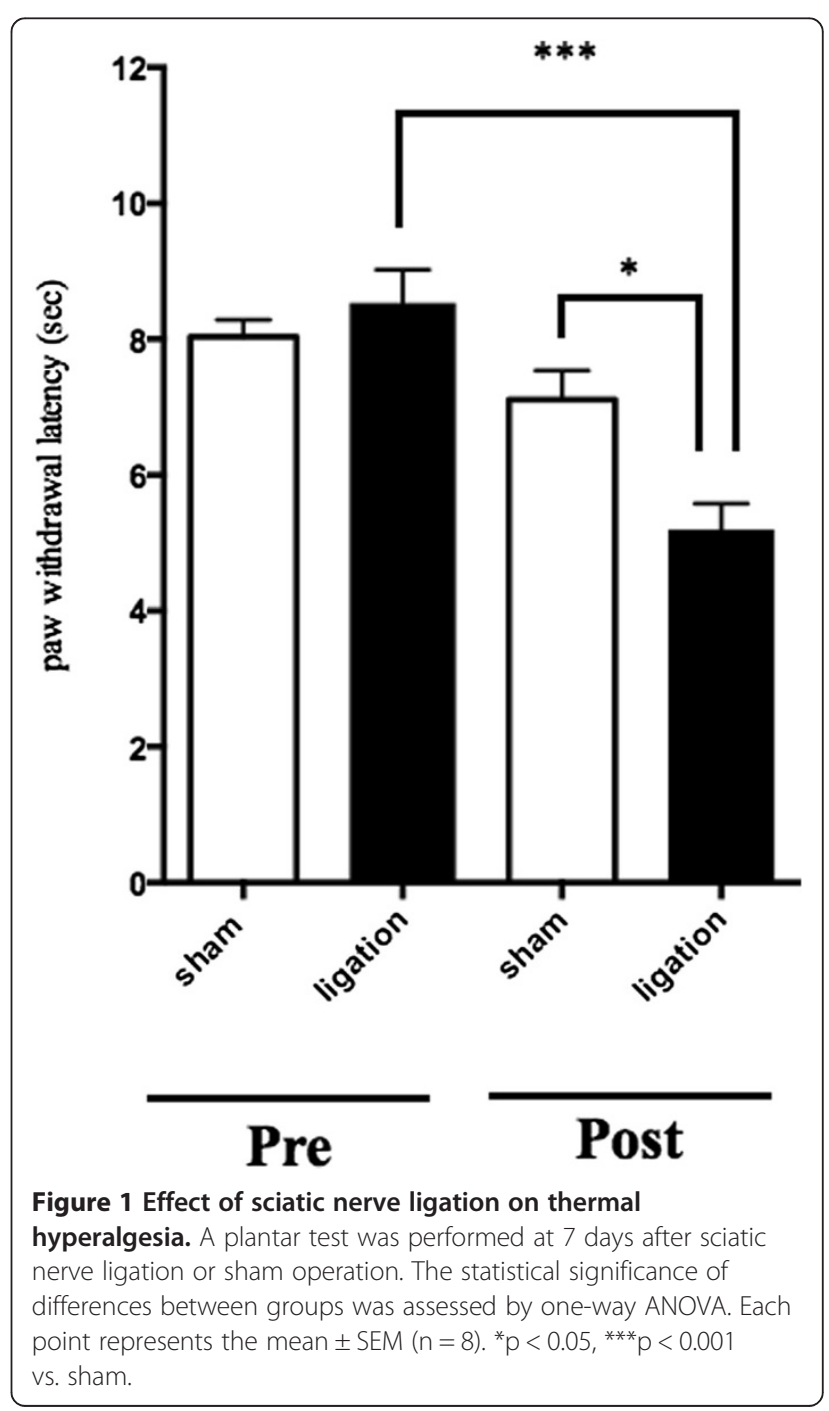



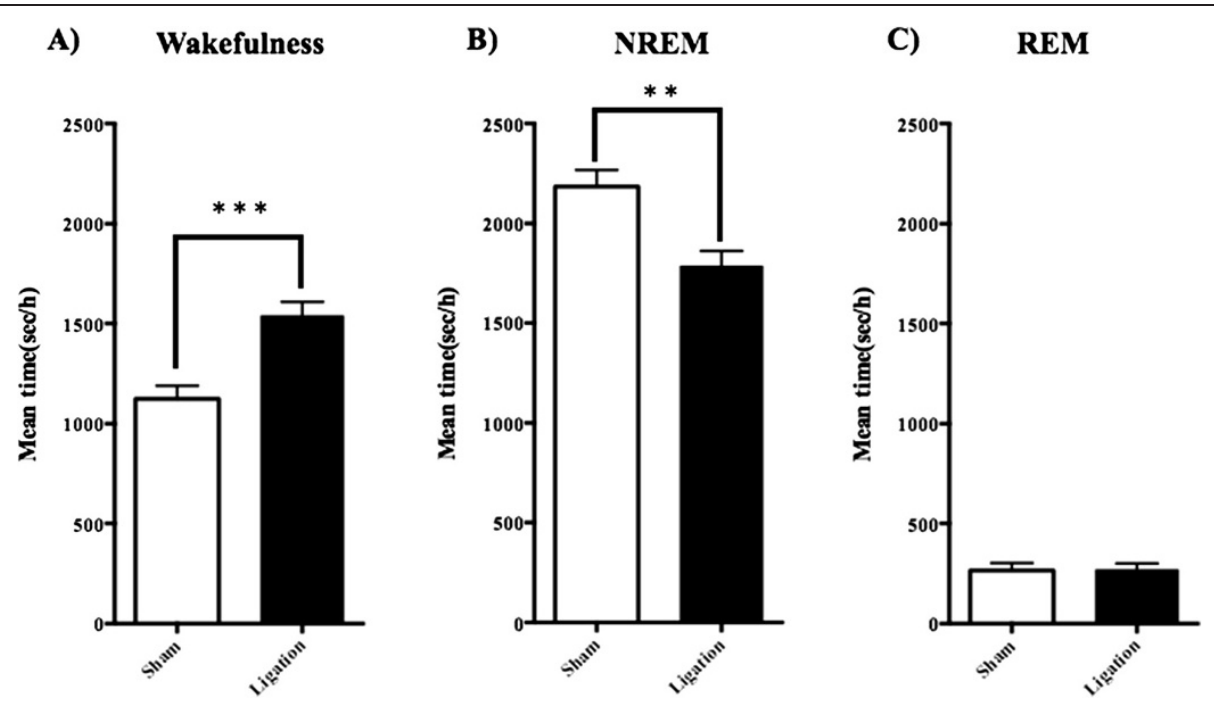

Figure $\mathbf{2}$ Change in the sleep/wake pattern as determined by EEG/EMG at $\mathbf{7}$ days after sciatic nerve ligation. The total mean times $(\mathrm{sec} / \mathrm{h})$ in wakefulness (A), NREM sleep (B) and REM sleep (C) were determined by EEG/EMG recording for 24 hrs. Each point represents the mean \pm SEM $(n=11)$. Student's $t$-test was performed. ${ }^{*} p<0.05$, ${ }^{* *} p<0.01$ and ${ }^{* * *} p<0.001$ compared with the sham group.

group). No difference in REM sleep was found between sham operation and sciatic nerve ligation for 7 days (Figure $2 \mathrm{C}$ ).

\section{Distribution of fluoro-gold(FG)-labeled cells following microinjection in the PFC}

To determine whether the DRN is linked to the PFC, which would permit possible functional influences on sleep and arousal neural circuits, we next investigated whether there were any neuronal projections from the DRN to the PFC using FG as a retrograde tracer. A schematic illustration of the injection site in the PFC is shown with a symbol (Figure 3A). Microinjection of FG into the region of the unilateral PFC produced a well-restricted injection site (Figure 3B). The DRN was shown to be located in a coronal section of the mouse brain (Figure 3C). Tryptophan hydroxylase 2 (Tph2)-labeled cells (Figure 3D, E) or FG-labeled cell bodies (Figure 3F) were apparently detected in the DRN after the microinjection of FG into the PFC. The population of retrogradely labeled neurons in the DRN also showed Tph2-immunoreactivity (Figure 3G).

\section{Change in the increase in dialysate 5-HT levels in the PFC} induced by electrical stimulation of the DRN

To investigate whether sciatic nerve ligation induces the hypersensitivity of DRN-PFC 5-HTergic neurons, we tested the effect of repetitive stimulation with rectangular electrical pulses for $15 \mathrm{~min}$. In an in vivo microdialysis study, the dialysate 5-HT levels in the PFC of sciatic nerve-ligated mice were significantly increased by electrical stimulation $(100 \mathrm{~Hz}, 150 \mu \mathrm{A})$ (Figure 4: $\mathrm{p}<0.01$ vs. sham group). Under the same conditions, there was no increase in the levels of dialysate 5-HT in the PFC of sham-operated mice.
Expression of optogenetic transgene in DRN and PFC neurons of Tph2-ChR2 transgenic mice

We investigated the expression of ChR2-EYFP in the DRN using immunohistochemistry. The DRN showed a high density of EYFP-immunoreactivity in slices of the mouse brainstem. To confirm the co-localization of ChR2-EYFP and Tph2 in the DRN, double-labeling experiments were performed. The results showed that Tph2-immunoreactivity was mostly co-localized with ChR2-EYFP (Figure 5).

Change in the dialysate levels of 5-HT induced by optical stimulation in the mouse DRN

We confirmed that optical stimulation of the DRN affected the levels of 5-HT secreted from nerve terminals in the PFC. In an in vivo microdialysis study, the dialysate 5-HT levels in the PFC of Tph2-ChR2 transgenic mice were significantly increased by optical stimulation of the DRN ( $<<0.05$ : vs. No stimuli, Figure 6).

\section{Optical stimulation of the DRN causes sleep-arousal transitions}

To determine whether neural activation in DRN-5HTergic neurons is necessary for interfering with sleep, we tested the effect of long-term optical stimulation of the DRN ( $8 \mathrm{~mW}, 20 \mathrm{~Hz}, 473 \mathrm{~nm}$, blue light for $1 \mathrm{hr}$ ) on the sleep-wake architecture during inactive periods. Tph2ChR2 transgenic mice showed a significant increase in wakefulness ( $p<0.01$ vs. No stimuli) and a significant decrease in NREM sleep ( $\mathrm{p}<0.01$ vs. No stimuli) during optical stimulation, compared with no stimuli (Figure 7A). The duration of NREM sleep episodes was significantly decreased during optical stimulation in these mice (Figure 7B: 


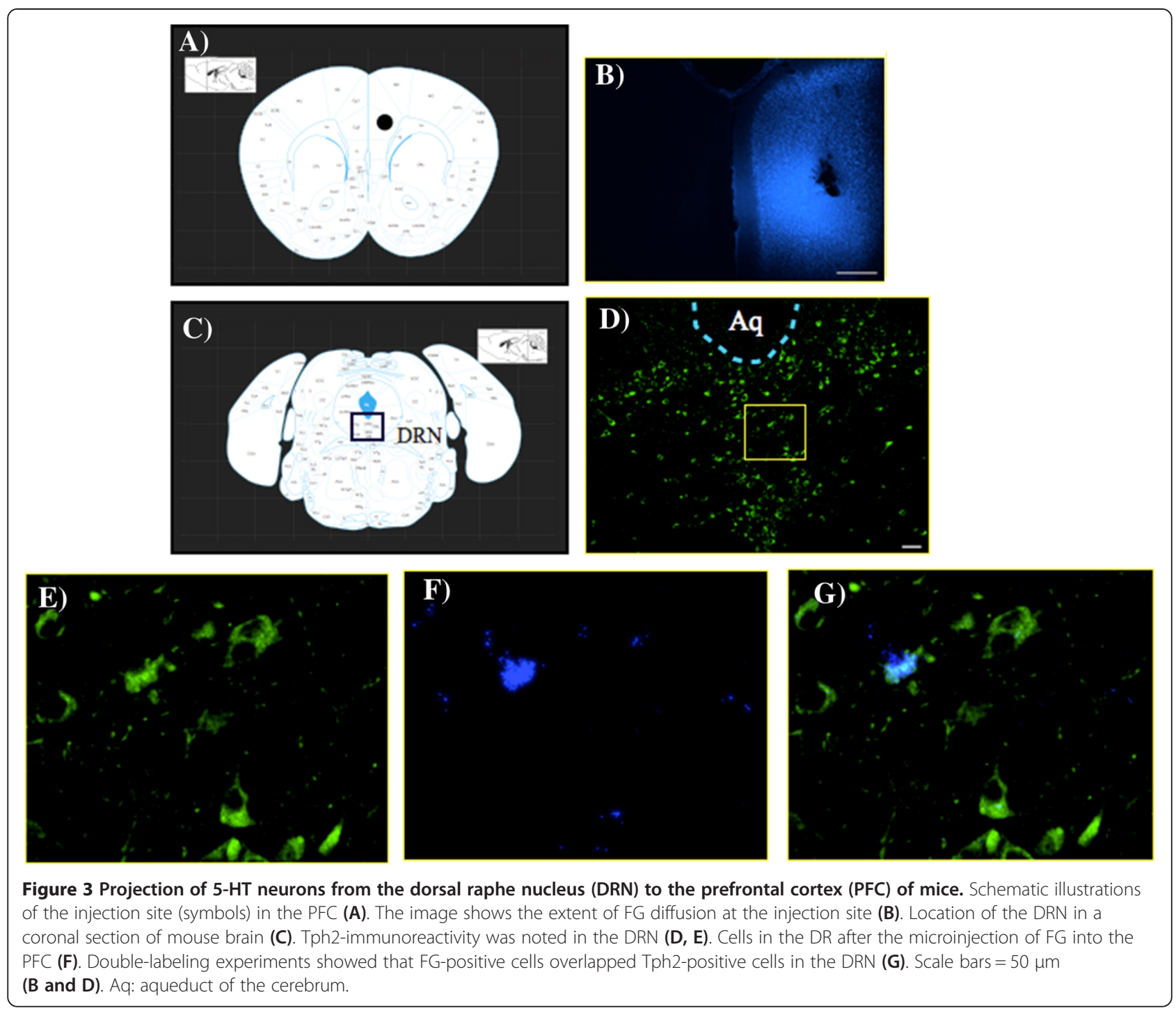

$\mathrm{p}<0.01$ vs. No stimuli). During optical stimulation, there was a significant decrease in slow wave activity $(0.5-4 \mathrm{~Hz})$ in Tph2-ChR2 transgenic mice $(\mathrm{p}<0.05$ : vs. No stimuli, Figure $7 \mathrm{C}$ ).

\section{Discussion}

Sciatic nerve constriction was previously reported to induce poor sleep quality in rats, particularly during the first week with this condition [24]. In our present study, we demonstrated that sciatic nerve-ligated mice exhibit dysregulation of the sleep/arousal pattern. These findings suggest that neuropathic pain induced by peripheral nerve injury may lead to the development of insomnia.

We analyzed the neural circuit using the retrograde tracer FG to investigate the nuclear origins of 5-HTergic neurons. We injected FG into the PFC, which is associated with sleep/arousal patterns [25]. FG-labeled cell bodies were detected in the DRN, and Tph2-immunoreactivity was revealed in a population of these labeled neurons. The DRN is one of the raphe nuclei located in the midline of the brainstem and is one of the largest 5-HTergic nuclei. Immunohistochemical studies have reported that the DRN contains as many as 9,200 5-HT neurons [26] or as few as 4,000 [27] in mice. Neurons from the DRN provide a substantial proportion of the 5-HT innervation to the forebrain through their widespread projections [28].

In a previous study, 5-HT transporter expression was shown to be significantly increased in the DRN of sciatic nerve-ligated rats [13]. However, few studies have investigated the direct influence of neuropathic pain on DRN 5-HTergic neurons. Therefore, we performed an in vivo microdialysis study to determine the activity of 5-HTergic neurons from the DRN to the PFC under a neuropathic pain-like state. The 5-HT level at the PFC was significantly increased in nerve-ligated mice compared with that in sham-operated mice after electrical stimulation of the 


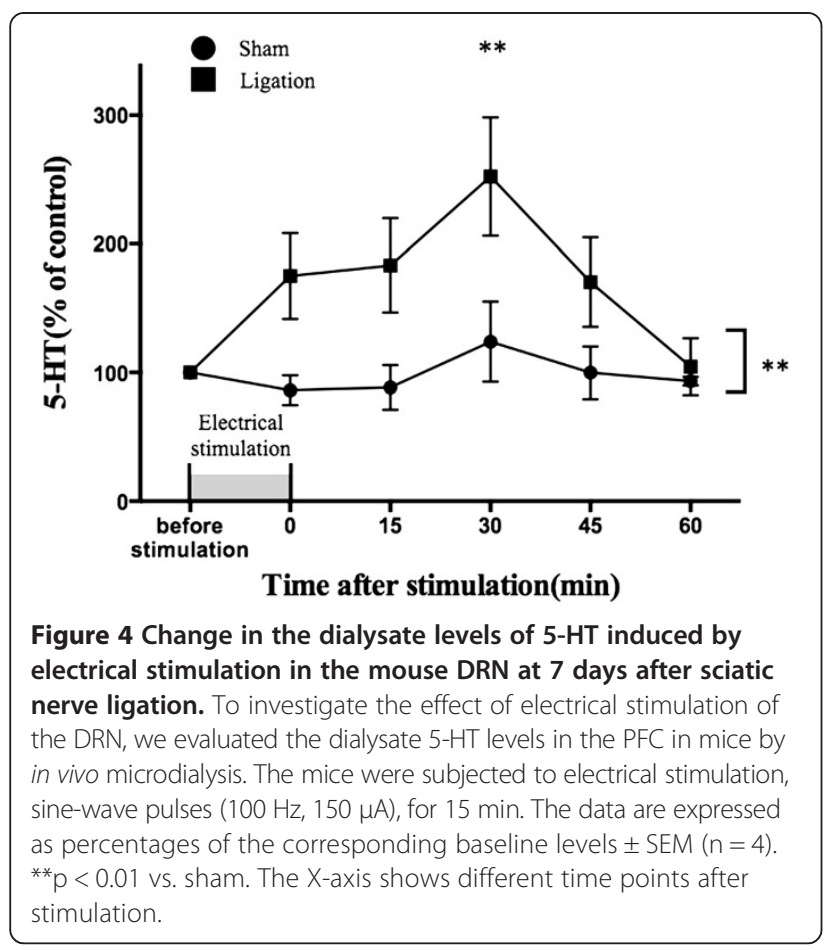

DRN. These results suggest that neuropathic pain is associated with an increased activity of 5-HTergic neurons projecting from the DRN to the PFC.

Several areas in the brainstem and forebrain are important for modulation and expression of the sleep/wake cycle. It is now well established that several neurotransmitters and neuropeptides are involved in modulation of the sleep/wake cycle. 5-HT has been known for many years to play a role in the modulation of sleep. Early studies suggested that 5 -HT is necessary to achieve and maintain behavioral sleep [3,29-31]. However, the 5-HTsleep connection was strongly contradicted by data on the activity of the DRN, in that the activity of DRN neurons was greatest during wakefulness, considerably lower during NREM sleep, and completely abolished during REM sleep [4-9]. Also, the cooling of raphe neurons induced sleep [32,33]. Thus, it is still very controversial how and where 5-HT participates in this modulation.

A major advantage of an optogenetic approach versus traditional electrical stimulation [34] or pharmacological manipulation [35-38] is its ability to elicit neural activity at specifically defined temporal windows with minimal disturbance to the animal [39]. Electrical stimulation has a high probability of exerting excessive effects on other neurons and glial cells around the target, since the DRN in mice is quite small. On the other hand, the stimulation of DRN neurons using the local microinjection of pharmacological agents affects circumventricular organs due to the anatomical location of the DRN. In fact, it is possible for the injected agents to leak into the cerebral ventricles. The optogenetic gain-of-function approach has achieved not only regiospecific effects but also efficient genetic targeting of 5-HTergic neurons, which may explain the difference between the results of the optogenetic approach and those of other studies. In the present study, we therefore performed a system function analysis of DRN-5-HTergic neurons using transgenic mice that express ChR2 in neurons containing Tph2. We first performed an immunohistochemistry study to evaluate the expression of ChR2 in 5-HTergic neurons in the DRN. Although our immunostaining experiment on DRN neurons in acute brainstem slices showed ChR2 expression

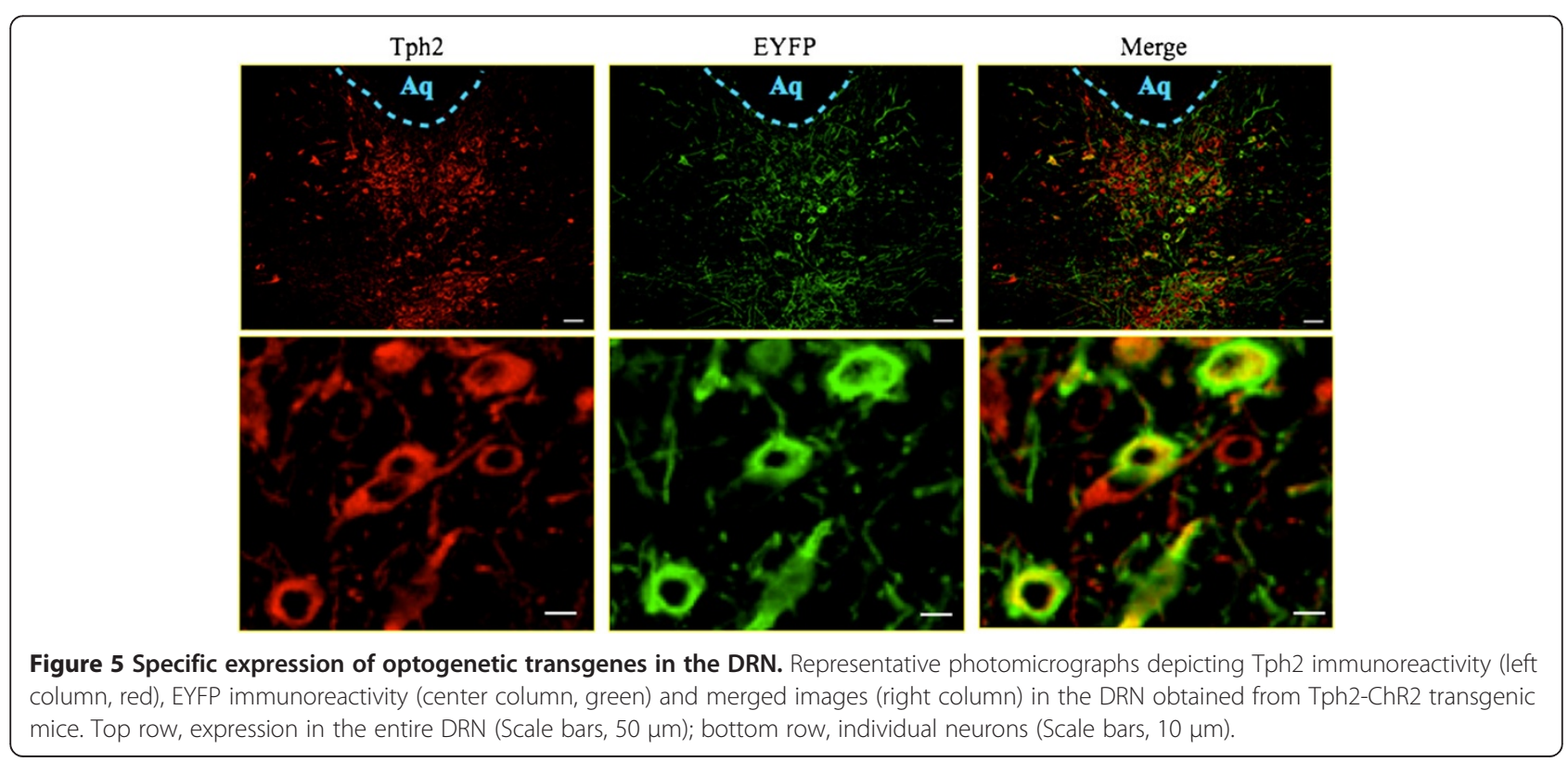




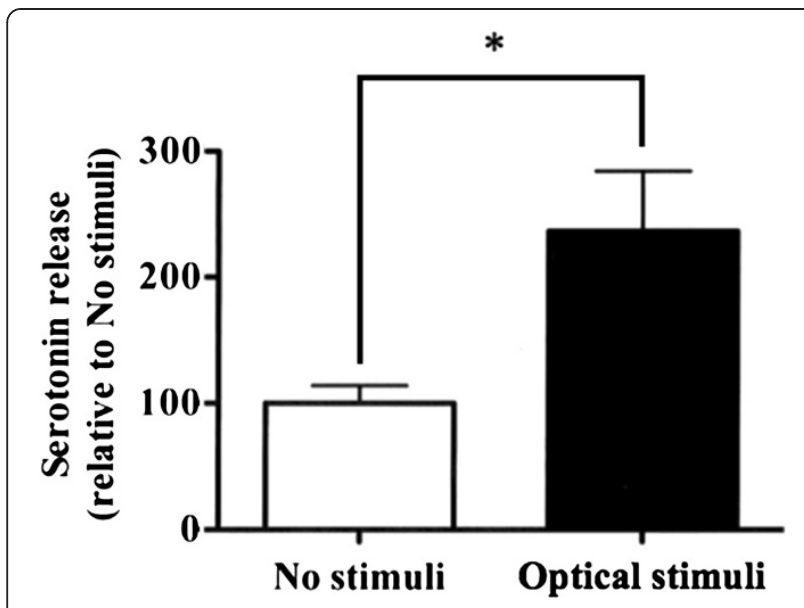

Figure 6 Change in the dialysate levels of $5-\mathrm{HT}$ induced by optical stimulation in the mouse DRN. Extracellular 5-HT content in the PFC during optical stimulation at $20 \mathrm{~Hz}$ in the DRN. Values are shown as the means with $\operatorname{SEM}(n=3) .{ }^{*} p<0.05$ vs. No stimuli.

in DRN 5-HTergic neurons, we cannot be certain that these tools modulate neural activity as precisely in vivo. Therefore, we performed an in vivo microdialysis study to evaluate the levels of 5-HT released in the PFC during optical stimulation of the DRN, which increases the 5HT level. Using this strategy, we confirmed the proper functioning of ChR2 expressed in the DRN. Next, we evaluated changes in the sleep and arousal pattern using EEG and EMG during optical stimulation of the DRN expressing ChR2. With optical stimulation, the total duration of wakefulness increased and that of NREM sleep decreased. These results suggest that the activation of
DRN 5-HTergic neurons facilitates awakening. In contrast, activation of the DRN caused a decrease in the duration of the NREM sleep stage. Therefore, we conclude that the DRN is sufficient to promote sleep-towake transitions. Furthermore, in the power density of the EEG analysis, there was a significant decrease in slow wave activity during optical stimulation. This observation indicates that the activation of DRN-5-HTergic neurons has the potential to change the quality of sleep.

The functional role of 5-HT neurons in relation to the sleep/wake cycle may not only be confined to the DRN, but is also evident in other 5-HT sources of the brainstem [40], such as the median raphe nucleus, which gives rise to the majority of serotonergic ascending fibers into the forebrain [41-43]. Thus, further experiments on the influence of other 5-HT sources are needed to better understand the role of 5-HTergic networks in regulating the sleep-wake pattern under both physiological and chronic pain conditions.

Based on genetic [44-48] and pharmacological experiments [36,49-52], it is accepted that 5-HT functions predominantly to promote wakefulness. Although we demonstrated that both neuropathic pain and the specific activation of DRN-5HTergic neurons lead to a decrease in NREM sleep and no difference of REM sleep, the causal mechanism of this difference between REM and NREM is still unclear. In addition, it is unclear whether the enhanced release of $5-\mathrm{HT}$ in the PFC is essential for neuropathic pain-induced sleep dysregulation, since our preliminary data show that no significant increase in the basal level of released 5-HT was likely to be seen without electrical stimulation when the sleep/wake pattern was
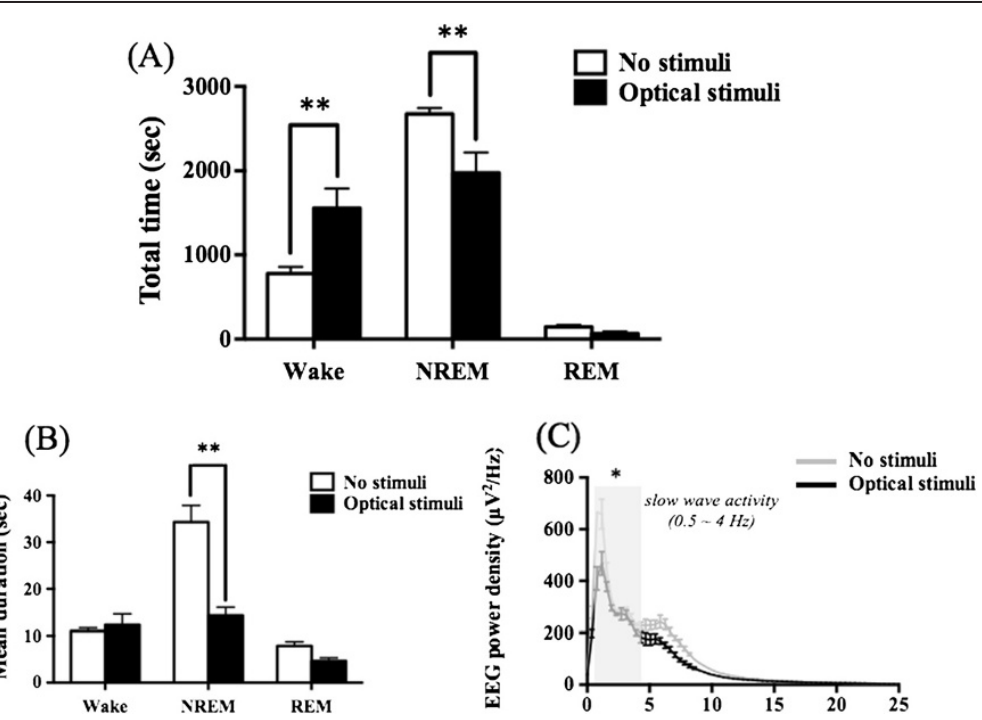

Figure 7 Changes in the sleep/wake pattern induced by optical stimulation in the mouse DRN. Mean time (A), mean duration (B) and relative EEG power of NREM (C) were determined by EEG/EMG recordings for $3 \mathrm{hr}$. Values are shown as the means with $\operatorname{SEM}(n=3)$. ${ }^{*} p<0.05$ and ${ }^{* *} p<0.01$ vs. No stimuli. 
changed (data not shown). We hypothesize that the reason why we did not observe an increase at this stage may be because under pathophysiological conditions, chronic pain may gradually increase the basal release of 5-HT in the PFC and affect the sleep/wake pattern, but constantly this will return to the normal level. Although we cannot completely exclude the possibility that the enhanced release of $5-\mathrm{HT}$ in the PFC is not essential for chronic pain-induced abnormal sleep, we propose here that this activation in DRN neurons may, at least in part, be associated with sleep dysregulation under a neuropathic pain-like state. Furthermore, we also believe that other nuclei, such as the noradrenergic locus ceruleus nucleus [53], histaminergic tuberomammilary nucleus [54], cholinergic pedunculopontine nucleus and lateral tegmental nucleus [55,56], as well as multiple cell types in the basal forebrain [57], may also presumably contribute to sleep-to-wake transitions.

\section{Conclusion}

In conclusion, we have demonstrated that neuropathic pain leads to sleep dysregulation, in parallel with the increased activity of DRN 5-HTergic neurons. Using an optogenetic approach, we found that the activation of DRN neurons facilitates sleep-to-wake transitions, which results in the dysregulation of sleep. Although further clarification is needed, we propose that acceleration of the activity of DRN-5-HTergic neurons may at least partly be associated with sleep dysregulation in a state of neuropathic pain.

\section{Methods}

\section{Animals}

The present study was conducted in accordance with the Guiding Principles for the Care and Use of Laboratory Animals at Hoshi University, as adopted by the Committee on Animal Research of Hoshi University (Tokyo, Japan). Male C57BL/6 J mice (8 weeks; Tokyo Laboratory Animals Science, Tokyo, Japan) and Tph2ChR2 transgenic mice (B6; SJL-TG (Tph2-COP*H134R/ EYFP) 5 Gfng/J) [16] were used in this study. Tph2 is the rate-limiting enzyme in the synthesis of $5-\mathrm{HT}$ in the central nervous system. The transgene ChR2 (H134R)EYFP was constructed into the ATG (initiation codon) site of the first exon of Tph2. Animals were housed in a room maintained at $23 \pm 1^{\circ} \mathrm{C}$ with a 12-hr light-dark cycle. Food and water were available ad libitum. Every effort was made to minimize the numbers and any suffering of animals used in the following experiments. Each animal was used only once.

\section{Neuropathic pain model}

Mice were anesthetized with $3 \%$ isoflurane. We produced a partial sciatic nerve ligation model as described previously [58]. In brief, the sciatic nerve on the right side (ipsilateral side) was tied by a tight ligature with 8-0 silk suture around approximately one-half its diameter under a light microscope (SD30; Olympus, Tokyo, Japan). In sham-operated mice, the nerve was only exposed without ligation.

\section{Measurement of thermal thresholds}

C57BL/6 J mice were used for the measurement of thermal thresholds. To assess the sensitivity to thermal stimulation, the right plantar surface of mice was tested individually by using a well-focused, radiant heat light source (model 33 Analgesia Meter; IITC/Life Science Instruments, California, USA). The intensity of the thermal stimulus was adjusted to achieve an average baseline paw-withdrawal latency of approximately $8-10 \mathrm{sec}$ in naive mice. The paw-withdrawal latency was determined as the average of three measurements per paw. Only quick hind paw movements (with or without licking of hind paws) away from the stimulus were considered to be a withdrawal response. Paw movements associated with locomotion or weight-shifting were not counted as a response. The paws were measured alternating between the left and right with an interval of more than 3 min between measurements. Before the behavioral responses to the thermal stimulus were tested, mice were habituated for at least $1 \mathrm{hr}$ in a clear acrylic cylinder (15 cm high and $8 \mathrm{~cm}$ in diameter). Under these conditions, the latency of paw withdrawal in response to the thermal stimulus was tested. The data represent the average value for the withdrawal latency of the right hind paw.

\section{EEG and EMG recordings}

Under 3\% isoflurane anesthesia, mice were implanted with EEG and EMG electrodes for polysomnographic recordings (Pinnacle Technology, Oregon, USA). Briefly, to monitor EEG signals, two stainless steel EEG recording screws were positioned $1 \mathrm{~mm}$ anterior to the bregma or lambda, both $1.5 \mathrm{~mm}$ lateral to the midline. EMG activity was monitored by stainless steel, Teflon-coated wires placed bilaterally into both trapezius muscles. Sleep-wake states were then monitored for a period of $24 \mathrm{hr}$ at 2 days after placement of the EEG recording screws, encompassing both the baseline and the experimental day. The EEG/ EMG signals were amplified, filtered (EEG, 0.5-30 Hz; EMG, 20-200 Hz), and digitized at a sampling rate of $128 \mathrm{~Hz}$. The collected data were analyzed by software (SLEEPSIGN; Kissei Comtec, Nagano, Japan). Vigilance was automatically classified off-line under 5 -sec epochs into three stages, i.e., wakefulness, REM and NREM sleep, by SLEEPSIGN according to the standard criteria. As a final step, defined sleep-wake stages were examined visually and corrected, if necessary. For each epoch, the EEG power density in the $\delta$ wave $(0.75-4.0 \mathrm{~Hz})$ and $\theta$ wave 
(6.25-9.0 Hz) and the integrated EMG value were displayed on a PC monitor. Three vigilance states -(1) waking (high EMG and low EEG amplitude and high $\theta$ wave activity concomitant with the highest EMG values), (2) NREM sleep (low EMG and high EEG amplitude, high $\delta$ wave activity), and (3) REM sleep (low EMG and low EEG amplitude, high $\theta$ wave activity)- were determined for 5-sec epochs, and the scores were entered into a computer via a keyboard. EEG and EMG activities were monitored at 7 days after sciatic nerve ligation.

\section{Retrograde tracing study}

First, C57BL/6 J mice were deeply anesthetized with $3 \%$ isoflurane. The anesthetized animals were placed in a stereotaxic apparatus. The skull was exposed, and a hole was drilled through the skull over the dorsolateral PFC, according to an atlas [59]. Micropipettes were filled with retrograde tracer, FG solution (Fluorochrome, Colorado, USA; $4 \%$ solution in saline), and $0.2 \mu \mathrm{l}$ of the tracer was injected over $30 \mathrm{sec}$. The micropipette was left in place for $1 \mathrm{~min}$ after injection was complete to avoid leakage of the tracer along the pipette track, and then withdrawn from the brain. Two days after the injections, animals were perfused with formalin. The distribution of goldemitting FG retrogradely labeled neurons and FG injection sites were detected using a microscope (BX-60; Olympus, Tokyo, Japan).

\section{Electrical stimulation}

At the start of the surgical procedures, C57BL/6 J mice were anesthetized with $3 \%$ isoflurane and placed in a small animal stereotaxic apparatus. Mice underwent surgical implantation of a bipolar concentric electrode (MS3033SPCE; Bio Research, Nagoya, Japan) at least 1 day before the experiments. The electrode was placed in the DRN (from the bregma: anteroposterior, $-4.9 \mathrm{~mm}$; mediolateral, $+0 \mathrm{~mm}$; dorsoventral, $-3.4 \mathrm{~mm}$ ) and fixed to the skull with quick self-curing acrylic resin. Electrical stimulation was controlled by an electronic stimulator (Nihon Kohden, Tokyo, Japan) to generate repetitive rectangular pulses (duration, $500 \mu$ s; intensity, $150 \mu \mathrm{A}$; frequency, $100 \mathrm{~Hz}$ ).

\section{In vivo microdialysis and high-performance liquid chromatography}

Under anesthesia with $3 \%$ isoflurane, mice were placed in a stereotaxic apparatus and a microdialysis probe (D-1-6-01; Eicom, Tokyo, Japan) was implanted directly into the PFC (from the bregma: anteroposterior, $+1.5 \mathrm{~mm}$; mediolateral, + $0.5 \mathrm{~mm}$; dorsoventral, $-3.7 \mathrm{~mm}$ ) according to an atlas of the mouse brain [59]. The probe was fixed to the cranial bone with quick self-curing acrylic resin. More than $24 \mathrm{hr}$ after surgery, the mice were placed in experimental cages. The probes were perfused continuously $(1 \mu \mathrm{l} / \mathrm{min})$ with artificial cerebrospinal fluid: $0.9 \mathrm{mM} \mathrm{MgCl}_{2}, 147.0 \mathrm{mM} \mathrm{NaCl}, 4.0 \mathrm{mM} \mathrm{KCl}$, and $1.2 \mathrm{mM} \mathrm{CaCl}_{2}$. Outflow fractions were collected every $15 \mathrm{~min}$. After more than four baseline fractions were collected, mice were given electrical stimulation or optical stimulation for $15 \mathrm{~min}$. Dialysis samples were collected for $1 \mathrm{hr}$ after electrical stimulation or optical stimulation and analyzed by high-performance liquid chromatography with electrochemical detection (HTEC-500; Eicom, Tokyo, Japan). 5-HT was separated by column chromatography and identified and quantified by the use of a standard (Sigma Aldrich, Missouri, USA).

\section{Immunohistochemistry}

Tph2-ChR2 transgenic mice were deeply anesthetized by the inhalation of $3 \%$ isoflurane with oxygen and perfusionfixed with $4 \%$ paraformaldehyde in $0.1 \mathrm{M}$ phosphatebuffered saline ( $\mathrm{pH}$ 7.4). After perfusion, the brain was quickly removed and thick coronal sections of the frontal cortex including the cingulate cortex or the brainstem including the DRN were rapidly dissected and fixed in $4 \%$ paraformaldehyde for $2 \mathrm{hr}$. They were then permeated with $20 \%$ sucrose in $0.1 \mathrm{M}$ phosphate-buffered saline for 1 day and 30\% sucrose in $0.1 \mathrm{M}$ phosphate-buffered saline for 2 days with agitation. The brain sections were finally frozen in an embedding compound (Sakura Finetechnical, Tokyo, Japan) on isopentane using liquid nitrogen and stored at $-30^{\circ} \mathrm{C}$. Transverse sections were cut with a cryostat (Leica CM1510; Leica Microsystems, Heidelberg, Germany) at a thickness of $8 \mu \mathrm{m}$ and thaw-mounted on poly-L-lysine-coated glass slides. Brain sections including PFC or DRN were incubated in blocking solution, 3\% normal goat serum (Vector Laboratories, California, USA) in $0.01 \mathrm{M}$ phosphate-buffered saline, for $1 \mathrm{hr}$ at room temperature, and then incubated for $48 \mathrm{hr}$ at $4^{\circ} \mathrm{C}$ with primary antibodies diluted in 3\% normal goat serum: antiTph2 (mouse monoclonal.; Sigma Aldrich, Missouri, USA) and anti-green fluorescent protein (GFP; rabbit polyclonal; Molecular Probes, Oregon, USA) for double-staining. The antibody was then rinsed with phosphate-buffered saline and incubated with an appropriate secondary antibody conjugated with Alexa $^{\mathrm{Tm}} 546$ (Molecular Probes, Oregon, USA) for $2 \mathrm{hr}$ at room temperature. Fluorescence of immunolabelling was detected using a light microscope (BX61; Olympus, Tokyo, Japan) and digitized images.

\section{Optical stimulation}

At the start of the surgical procedures, Tph2-ChR2 transgenic mice were anesthetized with $3 \%$ isoflurane and placed on a small animal stereotaxic apparatus. Mice underwent the surgical implantation of an $8 \mathrm{~mm}$ unilateral cannula (EIM-300; Eicom, Tokyo, Japan) at least 1 day before the experiments. The cannula was placed above the DRN and fixed to the skull with quick self-curing acrylic 
resin. An optical fiber $(500 \mu \mathrm{m}$ diameter; Lucir, Osaka, Japan) was placed inside the implanted cannula at least $2 \mathrm{hr}$ before the experiments. The light source was a $473 \mathrm{~nm}$ blue laser (COME2-LB473 model; Lucir, Osaka, Japan) controlled by an electronic stimulator (Nihon Kohden, Tokyo, Japan) to generate tonic light pulses (duration, $10 \mathrm{~ms}$; frequency, $20 \mathrm{~Hz}$; intensity, $9 \mathrm{~mW}$ ). The power output was measured at the tip of the fiber with a NOVA light meter (Ophir, Saitama, Japan) when the laser was activated in continuous mode.

\section{Statistical analysis}

Data are expressed as the mean with SEM. The statistical significance of differences between the groups was assessed with one-way ANOVA or two-way ANOVA followed by the Bonferroni multiple comparisons test or unpaired-Student's $t$ test as appropriate. All statistical analyses were performed with Prism version 5.0a or 6.0b (GraphPad Software, California, USA).

\author{
Abbreviations \\ ChR2: Channel rhodopsin 2; DRN: Dorsal raphe nucleus: \\ EEG: Electroencephalogram; EMG: Electromyogram; FG: Fluoro-gold; \\ GFP: Green fluorescent protein; NREM: Non rapid eye movement; \\ PFC: Prefrontal cortex; REM: Rapid eye movement; Tph2: Tryptophan \\ hydroxylase-2; 5-HT: 5-hydroxytryptamine (Serotonin).
}

\section{Competing interests}

The authors declare that they have no competing financial interests.

\section{Authors' contributions}

HI performed experiments, analyzed data and wrote the first draft of the manuscript. MY designed experiments, performed experiments and analyzed data. $\mathrm{AY}$ and $\mathrm{MN}$ designed and performed experiments. $\mathrm{CK}, \mathrm{AH}$ and $\mathrm{YS}$ performed experiments. DI, HS and MY wrote the paper. MN conceptualized the hypothesis, supervised research and finalized the manuscript. All authors read and approved the final manuscript.

\section{Acknowledgements}

This work was supported by a Grant-in-Aid for Scientific Research from the Ministry of Education, Culture, Sports, Science and Technology of Japan. The present study was conducted in accordance with the Guiding Principles for the Care and Use of Laboratory Animals, Hoshi University, as adopted by the Committee on Animal Research of Hoshi University.

\section{Author details}

'Department of Pharmacology, Hoshi University School of Pharmacy and Pharmaceutical Sciences, 2-4-41 Ebara, Shinagawa-ku, Tokyo 142-8501, Japan. ${ }^{2}$ Department of Anesthesiology, Graduate School of Medical and Pharmaceutical Sciences for Education, Toyama University, 2630 Sugitani, Toyama 930-0194, Japan.

Received: 3 November 2013 Accepted: 20 December 2013 Published: 26 December 2013

\section{References}

1. Smith MT, Perlis ML, Smith MS, Giles DE, Carmody TP: Sleep quality and presleep arousal in chronic pain. J Behav Med 2000, 23(1):1-13.

2. Morin CM, Gibson D, Wade J: Self-reported sleep and mood disturbance in chronic pain patients. Clin J Pain 1998, 14(4):311-314.

3. Jouvet $\mathrm{M}$ : The role of monoamines and acetylcholine-containing neurons in the regulation of the sleep-waking cycle. Ergeb Physiol 1972, 64:166-307.

4. Trulson ME, Jacobs BL: Raphe unit activity in freely moving cats: correlation with level of behavioral arousal. Brain Res 1979, 163(1):135-150.
5. Cespuglio R, Faradji H, Gomez ME, Jouvet M: Single unit recordings in the nuclei raphe dorsalis and magnus during the sleep-waking cycle of semi-chronic prepared cats. Neurosci Lett 1981, 24(2):133-138.

6. Lydic R, McCarley RW, Hobson JA: Serotonin neurons and sleep. II. Time course of dorsal raphe discharge, PGO waves, and behavioral states. Arch Ital Biol 1987, 126(1):1-28.

7. McGinty DJ, Harper RM: Dorsal raphe neurons: depression of firing during sleep in cats. Brain Res 1976, 101(3):569-575.

8. Puizillout JJ, Gaudin-Chazal G, Daszuta A, Seyfritz N, Ternaux JP: Release of endogenous serotonin from "encephale isole" cats. II - Correlations with raphe neuronal activity and sleep and wakefulness. J Physiol Paris 1979, 75(5):531-537.

9. Sakai K: Sleep-waking discharge profiles of dorsal raphe nucleus neurons in mice. Neuroscience 2011, 197:200-224.

10. Sanders KH, Klein CE, Mayor TE, Heym C, Handwerker HO: Differential effects of noxious and non-noxious input on neurones according to location in ventral periaqueductal grey or dorsal raphe nucleus. Brain Res 1980, 186(1):83-97.

11. Porro CA, Cavazzuti M, Galetti A, Sassatelli L: Functional activity mapping of the rat brainstem during formalin-induced noxious stimulation. Neuroscience 1991, 41(2-3):667-680

12. Dai JL, Zhu YH, Li XY, Huang DK, Xu SF: C-fos expression during electroacupuncture analgesia in rats-an immunohistochemical study. Acupunct Electrother Res 1992, 17(3):165-176.

13. Rojo ML, Rodriguez-Gaztelumendi A, Pazos A, Diaz A: Differential adaptive changes on serotonin and noradrenaline transporters in a rat model of peripheral neuropathic pain. Neurosci Lett 2012, 515(2):181-186.

14. Deisseroth K: Optogenetics. Nat Methods 2011, 8(1):26-29.

15. Deisseroth K, Feng G, Majewska AK, Miesenbock G, Ting A, Schnitzer MJ: Next-generation optical technologies for illuminating genetically targeted brain circuits. J Neurosci 2006, 26(41):10380-10386.

16. Zhao S, Ting JT, Atallah HE, Qiu L, Tan J, Gloss B, Augustine GJ, Deisseroth K, Luo M, Graybiel AM, et al: Cell type-specific channelrhodopsin-2 transgenic mice for optogenetic dissection of neural circuitry function. Nat Methods 2011, 8(9):745-752.

17. Boyden ES, Zhang F, Bamberg E, Nagel G, Deisseroth K: Millisecondtimescale, genetically targeted optical control of neural activity. Nat Neurosci 2005, 8(9):1263-1268.

18. Yizhar O, Fenno LE, Davidson TJ, Mogri M, Deisseroth K: Optogenetics in neural systems. Neuron 2011, 71(1):9-34.

19. Fenno L, Yizhar O, Deisseroth K: The development and application of optogenetics. Annu Rev Neurosci 2011, 34:389-412.

20. Zhang F, Vierock J, Yizhar O, Fenno LE, Tsunoda S, Kianianmomeni A, Prigge M, Berndt A, Cushman J, Polle J, et al: The microbial opsin family of optogenetic tools. Cell 2011, 147(7):1446-1457.

21. Aravanis AM, Wang $L P$, Zhang F, Meltzer $L A$, Mogri MZ, Schneider MB, Deisseroth K: An optical neural interface: in vivo control of rodent motor cortex with integrated fiberoptic and optogenetic technology. J Neural Eng 2007, 4(3):S143-S156.

22. Kravitz AV, Kreitzer AC: Optogenetic manipulation of neural circuitry in vivo. Curr Opin Neurobiol 2011, 21(3):433-439.

23. Lima SQ, Miesenbock G: Remote control of behavior through genetically targeted photostimulation of neurons. Cell 2005, 121(1):141-152.

24. Andersen ML, Tufik S: Sleep patterns over 21-day period in rats with chronic constriction of sciatic nerve. Brain Res 2003, 984(1-2):84-92.

25. Rueter $L E$, Jacobs $B L:$ Changes in forebrain serotonin at the light-dark transition: correlation with behaviour. Neuroreport 1996, 7(5):1107-1111.

26. Daszuta A, Portalier P: Distribution and quantification of 5-HT nerve cell bodies in the nucleus raphe dorsalis area of C57BL and BALBC mice. Relationship between anatomy and biochemistry. Brain Res 1985, 360(1-2):58-64.

27. Ishimura K, Takeuchi Y, Fujiwara K, Tominaga M, Yoshioka H, Sawada T: Quantitative analysis of the distribution of serotonin-immunoreactive cell bodies in the mouse brain. Neurosci Lett 1988, 91(3):265-270.

28. Vertes RP: A PHA-L analysis of ascending projections of the dorsal raphe nucleus in the rat. J Comp Neurol 1991, 313(4):643-668.

29. Koella WP, Czicman J: Mechanism of the EEG-synchronizing action of serotonin. Am J Physiol 1966, 211(4):926-934.

30. Ursin R: Does para-chlorophenylalanine produce disturbed waking, disturbed sleep or activation by ponto-geniculo-occipital waves in cats? Waking Sleeping 1980, 4(3):211-221. 
31. Ursin R: Differential effect of para-chlorophenylalanine on the two slow wave sleep stages in the cat. Acta Physio/ Scand 1972, 86(2):278-285.

32. Cespuglio R, Gomez ME, Walker E, Jouvet M: Effect of cooling and electrical stimulation of nuclei of raphe system on states of alertness in cat. Electroencephalogr Clin Neurophysio/ 1979, 47(3):289-308.

33. Cespuglio R, Walker E, Gomez ME, Musolino R: Cooling of the nucleus raphe dorsalis induces sleep in the cat. Neurosci Lett 1976, 3(4):221-227.

34. Jha SK, Ross RJ, Morrison AR: Sleep-related neurons in the central nucleus of the amygdala of rats and their modulation by the dorsal raphe nucleus. Physiol Behav 2005, 86(4):415-426.

35. Portas CM, Thakkar M, Rainnie D, McCarley RW: Microdialysis perfusion of 8-hydroxy-2-(di-n-propylamino)tetralin (8-OH-DPAT) in the dorsal raphe nucleus decreases serotonin release and increases rapid eye movement sleep in the freely moving cat. J Neurosci 1996, 16(8):2820-2828.

36. Monti JM, Jantos H: Differential effects of the 5-HT1A receptor agonist flesinoxan given locally or systemically on REM sleep in the rat. Eur $\mathrm{J}$ Pharmacol 2003, 478(2-3):121-130.

37. Bradley PB, Marley E: Effect of tryptamine and tryptamine homologues on cerebral electrical activity and behaviour in the cat. Br J Pharmacol Chemother 1965, 24:659-674.

38. Adrien J, Tissier MH, Lanfumey L, Haj-Dahmane S, Jolas T, Franc B, Hamon M: Central action of 5-HT3 receptor ligands in the regulation of sleepwakefulness and raphe neuronal activity in the rat. Neuropharmacology 1992, 31(6):519-529.

39. Tye KM, Deisseroth K: Optogenetic investigation of neural circuits underlying brain disease in animal models. Nat Rev Neurosci 2012, 13(4):251-266.

40. Portas CM, Bjorvatn B, Ursin R: Serotonin and the sleep/wake cycle: special emphasis on microdialysis studies. Prog Neurobiol 2000, 60(1):13-35.

41. Jacobs BL, Fornal CA: Activity of brain serotonergic neurons in the behaving animal. Pharmacol Rev 1991, 43(4):563-578.

42. Adell A, Celada P, Abellan MT, Artigas F: Origin and functional role of the extracellular serotonin in the midbrain raphe nuclei. Brain Res Brain Res Rev 2002, 39(2-3):154-180.

43. Lechin F, van der Dijs B, Hernandez-Adrian G: Dorsal raphe vs. median raphe serotonergic antagonism. Anatomical, physiological, behavioral, neuroendocrinological, neuropharmacological and clinical evidences: relevance for neuropharmacological therapy. Prog Neuropsychopharmacol Biol Psychiatry 2006, 30(4):565-585.

44. Boutrel B, Franc B, Hen R, Hamon M, Adrien J: Key role of 5-HT1B receptors in the regulation of paradoxical sleep as evidenced in 5-HT1B knock-out mice. J Neurosci 1999, 19(8):3204-3212.

45. Boutrel B, Monaca C, Hen R, Hamon M, Adrien J: Involvement of 5-HT1A receptors in homeostatic and stress-induced adaptive regulations of paradoxical sleep: studies in 5-HT1A knock-out mice. J Neurosci 2002, 22(11):4686-4692.

46. Frank MG, Stryker MP, Tecott LH: Sleep and sleep homeostasis in mice lacking the 5-HT2C receptor. Neuropsychopharmacology 2002, 27(5):869-873.

47. Popa D, Lena C, Fabre V, Prenat C, Gingrich J, Escourrou P, Hamon M, Adrien J: Contribution of 5-HT2 receptor subtypes to sleep-wakefulness and respiratory control, and functional adaptations in knock-out mice lacking 5-HT2A receptors. J Neurosci 2005, 25(49):11231-11238.

48. Hedlund PB, Huitron-Resendiz S, Henriksen SJ, Sutcliffe JG: 5-HT7 receptor inhibition and inactivation induce antidepressantlike behavior and sleep pattern. Biol Psychiatry 2005, 58(10):831-837.

49. Monti $\mathrm{JM}$, Jantos $\mathrm{H}$ : Effects of the serotonin $5-\mathrm{HT} 2 \mathrm{~A} / 2 \mathrm{C}$ receptor agonist $\mathrm{DOI}$ and of the selective $5-\mathrm{HT} 2 \mathrm{~A}$ or $5-\mathrm{HT} 2 \mathrm{C}$ receptor antagonists EMD 281014 and SB-243213, respectively, on sleep and waking in the rat. Eur J Pharmacol 2006, 553(1-3):163-170.

50. Monti JM, Jantos H, Lagos P: Activation of serotonin 5-HT(1B) receptor in the dorsal raphe nucleus affects REM sleep in the rat. Behav Brain Res 2010, 206(1):8-16.

51. Monti JM, Jantos H, Monti D: Increased REM sleep after intra-dorsal raphe nucleus injection of flesinoxan or 8-OHDPAT: prevention with WAY 100635. Eur Neuropsychopharmacol 2002, 12(1):47-55.

52. Bjorvatn B, Ursin R: Effects of the selective 5-HT1B agonist, CGS 12066B, on sleep/waking stages and EEG power spectrum in rats. J Sleep Res 1994, 3(2):97-105.

53. Berridge CW, Waterhouse BD: The locus coeruleus-noradrenergic system: modulation of behavioral state and state-dependent cognitive processes. Brain Res Brain Res Rev 2003, 42(1):33-84.
54. Parmentier R, Ohtsu H, Djebbara-Hannas Z, Valatx JL, Watanabe T, Lin JS: Anatomical, physiological, and pharmacological characteristics of histidine decarboxylase knock-out mice: evidence for the role of brain histamine in behavioral and sleep-wake control. J Neurosci 2002, 22(17):7695-7711.

55. Steriade M: Acetylcholine systems and rhythmic activities during the waking-sleep cycle. Prog Brain Res 2004, 145:179-196.

56. Boucetta S, Jones BE: Activity profiles of cholinergic and intermingled GABAergic and putative glutamatergic neurons in the pontomesencephalic tegmentum of urethane-anesthetized rats. J Neurosci 2009, 29(14):4664-4674.

57. Hassani OK, Lee MG, Henny P, Jones BE: Discharge profiles of identified GABAergic in comparison to cholinergic and putative glutamatergic basal forebrain neurons across the sleep-wake cycle. J Neurosci 2009, 29(38):11828-11840.

58. Malmberg AB, Chen $C$, Tonegawa S, Basbaum Al: Preserved acute pain and reduced neuropathic pain in mice lacking PKCgamma. Science 1997, 278(5336):279-283

59. Franklin K, Paxinos G: The Mouse Brain in Stereotaxic Coordinates. San Diego: Academic; 2007

doi:10.1186/1756-6606-6-59

Cite this article as: Ito et al:: Analysis of sleep disorders under pain using an optogenetic tool: possible involvement of the activation of dorsal raphe nucleus-serotonergic neurons. Molecular Brain 2013 6:59.

\section{Submit your next manuscript to BioMed Central and take full advantage of:}

- Convenient online submission

- Thorough peer review

- No space constraints or color figure charges

- Immediate publication on acceptance

- Inclusion in PubMed, CAS, Scopus and Google Scholar

- Research which is freely available for redistribution 